\title{
Um estudo sobre o uso de dispositivos móveis e aplicações de aprendizagem móvel com foco em usuários idosos
}

\author{
Camila Dias de Oliveira ${ }^{1}$, Renata Pontin de Mattos Fortes ${ }^{1}$, Ellen Francine Barbosa ${ }^{1}$ \\ ${ }^{1}$ Instituto de Ciências Matemáticas e de Computação (ICMC-USP) \\ Caixa Postal 668 - CEP 13560-970 - São Carlos (SP), Brasil \\ camila_oliveira@usp.br, \{renata,francine\}@icmc.usp.br
}

\begin{abstract}
This paper presents a study on the use of mobile devices and mobile learning applications for elderly users. A questionnaire was answered by $28 \mathrm{el}$ derly users, which was complemented by an interview with two experts on older users' teaching. The results pointed out a considerable interest of these learners in mobile learning applications and indicated the importance of developing this type of application regarding to specific characteristics, giving special attention to the need of being intuitive. From these results, we proposed Crossword Learning, an application that works with teaching and learning aspects of the elderly through crosswords.
\end{abstract}

Resumo. Este artigo apresenta um estudo sobre o uso de dispositivos móveis e aplicações de aprendizagem móvel no contexto de usuários idosos. No estudo foi aplicado um questionário para 28 usuários idosos, o qual foi complementado por meio da realização de entrevistas com dois especialistas no ensino dos usuários mais velhos. Os resultados apontam um considerável interesse desses aprendizes em aplicações de aprendizagem móvel e indicam a importância de se desenvolver esse tipo de aplicação com características específicas, dando especial atenção à necessidade de serem intuitivas. A partir desses resultados, foi proposta, ainda, a Crossword Learning, aplicação que trabalha com aspectos de ensino e aprendizagem dos idosos por meio de palavras cruzadas.

\section{Introdução}

Segundo a Organização Mundial da Saúde (OMS), a expectativa de vida mundial teve um aumento de 5 anos no período de 2000 a 2015; ainda, a proporção de pessoas com 60 anos ou mais atingirá os 2 bilhões em 2050 [Organization et al. 2016]. No Brasil, esse cenário não é diferente; a expectativa de vida subiu mais de 30 anos no período de 1940 a 2016 [Marli 2017]. Além disso, estima-se que o número de pessoas com 65 anos ou mais irá quadruplicar até 2060 , atingindo um percentual de $26,8 \%$ da população brasileira [IBGE 2010].

Com o aumento da expectativa de vida e do número de idosos, tem-se uma heterogeneidade desse segmento que extrapola a faixa etária [Camarano et al. 2004]. Essas pessoas têm inserções diferentes na vida social e econômica do país, por conta de sua trajetória e experiências de vida. Diante dessa heterogeneidade, as demandas passam a ser diferenciadas, aumentando cada vez mais a necessidade de políticas públicas relacionadas à população idosa. 
VII Congresso Brasileiro de Informática na Educação (CBIE 2018)

Anais do XXIX Simpósio Brasileiro de Informática na Educação (SBIE 2018)

No Brasil, a legislação que busca regulamentar os direitos assegurados às pessoas com idade igual ou superior a 60 anos denomina-se Estatuto do Idoso [Brasil 2003]. Esse estatuto prevê que a família, a comunidade e a sociedade têm a obrigação de assegurar ao idoso o "direito à vida, à saúde, à alimentação, à educação, à cultura, ao esporte, ao lazer, ao trabalho, à cidadania, à liberdade, à dignidade, ao respeito e à convivência familiar e comunitária". Um ponto importante do Estatuto do Idoso diz respeito ao direito à educação. Em seu Capítulo V, Art. 21, tem-se que "os cursos especiais para idosos incluirão conteúdo relativo às técnicas de comunicação, computação e demais avanços tecnológicos, para sua integração à vida moderna" [Brasil 2003].

Diante desse contexto, tem-se a possibilidade de educação formal ou informal, a qual, ainda, pode ocorrer de forma presencial ou a distância. Emerge, assim, o conceito de aprendizagem móvel - mobile learning ou, simplesmente, m-learning. De forma geral, a aprendizagem móvel envolve a utilização da tecnologia móvel, isoladamente ou em conjunto com outras Tecnologias de Informação e Comunicação (TICs), para permitir a aprendizagem a qualquer hora e em qualquer lugar [Kraut 2013]. Além disso, a aprendizagem móvel é alimentada pela confluência de três fluxos tecnológicos [O'Malley et al. 2005]: o poder da computação, da comunicação e do desenvolvimento de interfaces inteligentes.

Essa modalidade de ensino tem gerado grande expectativa por parte de educadores e aprendizes, pois apresenta benefícios relacionados à flexibilidade de ensino e treinamento, uma vez que possibilita a adaptação com respeito ao espaço e ao tempo de utilização [Sarrab 2015, Sharples et al. 2009, Kukulska-Hulme and Sharples 2016]. A partir desse potencial de flexibilidade e adaptação, a aprendizagem móvel tem como um de seus objetivos democratizar o acesso à educação e, desse modo, deve ser adequada e estruturada para atender a população em geral, incluindo-se aqui as pessoas idosas. Considerando este público-alvo, faz-se necessário o desenvolvimento de aplicações de aprendizagem móvel acessíveis, uma vez que esses usuários podem ter habilidades distintas das que usualmente são comuns aos jovens estudantes. De fato, pessoas idosas podem ter suas capacidades físicas, sensoriais, de percepção e cognitiva comprometidas devido ao processo de envelhecimento [Whitbourne 2008]. Assim, o processo de aprendizado se torna diferenciado [Hanson 2010].

O termo acessibilidade pode ser definido em diversos contextos. Segundo a ISO 20282-2 [ISO 2013], acessibilidade é a "a medida em que um produto pode ser usado com eficácia, eficiência e satisfação por pessoas de uma população com a mais ampla gama de características e capacidades para alcançar um objetivo especificado em um contexto específico de uso". No contexto de "acessibilidade móvel", pode-se dizer que assegurar acessibilidade refere-se a tornar sites e aplicativos mais acessíveis para pessoas com deficiência (ou com algum tipo de limitação temporária), ao utilizarem telefones celulares e outros dispositivos móveis [W3C 2017].

Para que as aplicações de aprendizagem móvel (m-learning apps) sejam acessíveis e usáveis para diversas pessoas, é essencial conhecer o perfil e características do seu público-alvo [Dix et al. 2003, Lazar et al. 2010]. Assim, este artigo tem como objetivo apresentar dados obtidos por meio de instrumentos científicos de levantamento (questionários e entrevistas) e analisá-los, possibilitando o delineamento desse perfil específico de usuários aprendizes, os idosos. A partir dessas informações, pretende-se obter 
VII Congresso Brasileiro de Informática na Educação (CBIE 2018)

Anais do XXIX Simpósio Brasileiro de Informática na Educação (SBIE 2018)

parâmetros para criação de m-learning apps com foco em usuários idosos. Além disso, os resultados obtidos visam contribuir para o estabelecimento de um conjunto de diretrizes de acessibilidade que auxilie no processo de desenvolvimento de aplicações desse tipo.

No estudo realizado foi aplicado um questionário para 28 usuários idosos, o qual foi complementado por meio da realização de entrevista com dois especialistas no ensino dos usuários mais velhos. Os resultados indicaram que os idosos possuem interesse em aprender por meio de m-learning apps. Ademais, foi envidenciada uma expectativa de que a interface dessas aplicações fosse o mais intuitiva possível. A partir desses resultados, foi proposta uma m-learning app, denominada Crossword Learning [Oliveira et al. 2018]. A aplicação, ainda em fase de prototipação, visa ensinar os idosos sobre diferentes conteúdos, trabalhando com aspectos de ensino e aprendizagem por meio de palavras cruzadas.

Este artigo está organizado como segue. A Seção 2 apresenta os trabalhos relacionados à temática desse estudo. A Seção 3 aborda os procedimentos metodológicos utilizados para alcançar o objetivo principal do trabalho, descrevendo a aplicação do questionário e as entrevistas realizadas. A Seção 4 sintetiza os resultados obtidos e as principais considerações dos participantes na coleta de dados. A Seção 5 apresenta o protótipo de uma m-learning app, a Crossword Learning e suas principais funcionalidades. Finalmente, a Seção 6 sumariza as conclusões do estudo bem como as perspectivas para trabalhos futuros.

\section{Trabalhos relacionados}

Vários são os estudos que investigam o uso de dispositivos e aplicações móveis por usuários idosos. O estudo de [Kurniawan et al. 2006] procurou responder de que forma os atuais designs de telefones celulares podem ser melhorados para ajudar pessoas mais velhas. Os autores concluíram que o interesse dos idosos pelo uso de aplicativos móveis é subestimado e que, apesar de relatarem vários problemas de interação, os idosos conseguem realizar diversas tarefas em tais dispositivos.

Quanto ao uso de aplicações móveis, percebe-se que muitos são os aplicativos desenvolvidos para auxiliar o idoso com relação a saúde e melhor qualidade de vida, tal como apresentam [Boulos et al. 2014, Silva et al. 2015]. Seguindo esse contexto, [Zimmer et al. 2013] detalham o desenvolvimento de uma aplicação que buscou maximizar os níveis de desenvolvimento cognitivo do usuário idoso, por meio de um treinamento para a memória.

Com foco na aprendizagem móvel (m-learning), no trabalho de [Wang et al. 2009] foram investigados os fatores que afetam o aprendizado de uma pessoa. Os dados foram coletados com 330 pessoas e, dentre os resultados obtidos, observou-se que as diferenças de idade e de gênero influenciam na intenção de uso de aprendizagem móvel.

Apesar da variedade das pesquisas existentes, é importante notar que os trabalhos conduzidos possuem um foco diferente em relação ao presente trabalho. A pesquisa aqui descrita tem como objetivo compreender os interesses do perfil dos idosos em $m$-learning apps, de modo a contribuir para o desenvolvimento de softwares (aplicações) de aprendizagem móvel acessíveis aos idosos. 
VII Congresso Brasileiro de Informática na Educação (CBIE 2018)

Anais do XXIX Simpósio Brasileiro de Informática na Educação (SBIE 2018)

\section{Procedimentos metodológicos}

Esta investigação foi realizada visando essencialmente processar uma coleta de dados. De acordo com [Gerhardt and Souza 2009], em uma coleta de dados é importante não somente coletar informações que dêem conta dos conceitos (por meio de indicadores), mas também obter essas informações de forma que se possa aplicar posteriormente o tratamento necessário para testar as hipóteses. Portanto, é necessário antecipar, ou seja, preocupar-se, desde a concepção do instrumento, com o tipo de informação que ele permitirá fornecer e com o tipo de análise que deverá ser feita posteriormente.

Neste trabalho foram utilizados como instrumentos para a coleta de dados um questionário $^{1}$ e uma entrevista semi-estruturada ${ }^{2}$. Para ambos, procurou-se criar questões baseadas em cada um dos objetivos da pesquisa, conforme sugerido por [Gil 2002]. O desenvolvimento do questionário e da entrevista, bem como a caracterização dos participantes da pesquisa, são descritos a seguir.

\subsection{Desenvolvimento do questionário e da entrevista}

Este estudo foi realizado com o objetivo de auxiliar os desenvolvedores na implementação de m-learning apps acessíveis para pessoas idosas. Deste modo, o estudo foi dividido em duas etapas: (i) aplicação de questionário para usuários idosos; (ii) entrevista com especialistas no ensino de idosos.

As 19 questões contempladas pelo questionário abordavam características gerais do usuário, sobre o uso de dispositivos móveis e sobre o interesse dos usuários em $\mathrm{m}$ learning apps. A coleta de dados foi realizada presencialmente no mês de outubro de 2017, durante as aulas de Informática Básica para Teceira Idade, iniciativa oferecida pelo Instituto de Ciências Matemáticas e de Computação (ICMC) da Universidade de São Paulo (USP) - São Carlos. Como mecanismo de apoio para o desenvolvimento e aplicação do questionário, foi utilizado o Google Forms ${ }^{3}$, ferramenta online. Assim, durante as aulas, foi solicitado aos alunos acessar o link e responder às questões, sempre com o auxílio de monitores. No total, 28 pessoas, com idade superior a 50 anos, responderam ao questionário. Apesar de o estudo ser voltado para idosos (segundo o Estatuto do Idoso [Brasil 2003], idosos são quem tem mais do que 60 anos), 3 (três) participantes da pesquisa (idades 50, 56 e 57 anos) não eram qualificados como idosos, mas também foram considerados, por estarem engajados em atividades externas, regularmente, e comuns aos colegas idosos. O tempo de resposta variou de 15 minutos a 01 hora, dependendo da experiência do usuário com o uso da ferramenta de questionário online.

Já a entrevista semi-estruturada foi realizada com dois especialistas, que atuam como instrutores do curso de Práticas de Tablets ou Smartphones para Terceira Idade que faz parte do programa Universidade Aberta da Terceira Idade, também oferecido pelo ICMC/USP-São Carlos. A entrevista foi composta por oito questões abertas e ocorreu presencialmente, tendo duração de cerca de 20 minutos por especialista. Ela foi gravada e transcrita para facilitar a análise dos dados.

Vale destacar que tanto no questionário quanto na entrevista, os participantes responderam a um Termo de Consentimento Livre e Esclarecido (TCLE), declarando o inte-

\footnotetext{
${ }^{1}$ shorturl.at/psGT0

${ }^{2}$ shorturl.at/pzOY6

${ }^{3}$ https://www.google.com/forms
} 
VII Congresso Brasileiro de Informática na Educação (CBIE 2018)

Anais do XXIX Simpósio Brasileiro de Informática na Educação (SBIE 2018)

resse e aceitando participar da pesquisa. Todos eram cientes de que, a qualquer momento, poderiam desistir de continuar a participar.

\section{Resultados e análises}

\subsection{Questionário aplicado com os usuários}

Conforme dito anteriormente, o questionário foi respondido por pessoas com idade entre 50 a 81 anos. Dos 28 participantes, $14(50 \%)$ eram homens e $14(50 \%)$ mulheres. A maioria $(86 \%)$ possuia algum dispositivo móvel; dentre os dispositivos utilizados estão: (i) Smartphones e celulares (92\%); (ii) Tablets (4\%); (iii) Notebook (4\%).

Do total, 15 participantes (63\%) indicaram utilizar os dispositivos móveis diariamente (Figura 1), sendo que as atividades realizadas com esses dispositivos são diversas (Figura 2), com especial atenção ao uso dos dispositivos para fazer ligações $(60,71 \%)$ e acessar a Internet $(57,14 \%)$.
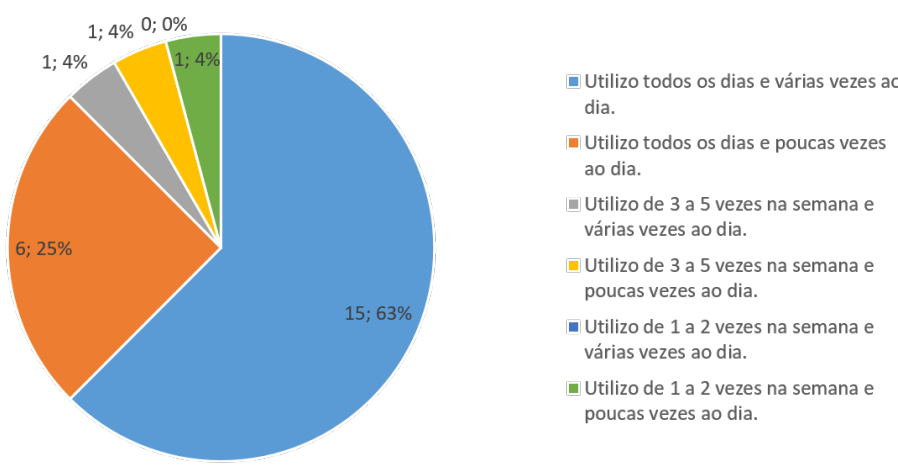

Figura 1. Tempo de uso do dispositivo móvel

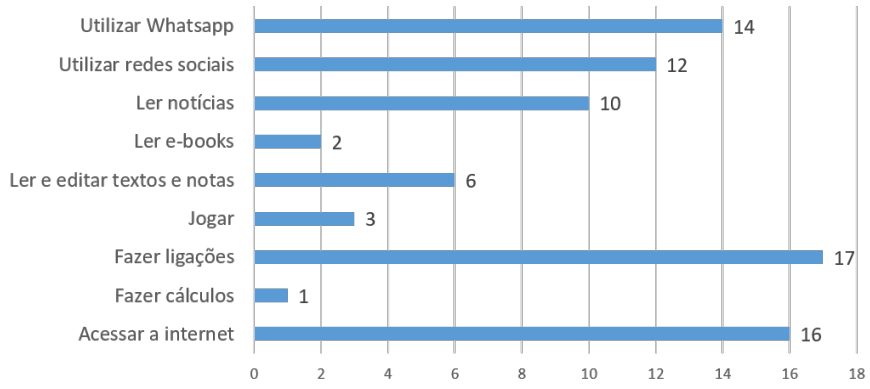

Figura 2. Atividades realizadas com o dispositivo móvel

A maioria dos participantes $(96,42 \%)$ também afirmou acreditar que os dispositivos móveis podem auxiliar as pessoas em seu dia-a-dia. Segundo comentários do questionário, esses dispositivos podem auxiliar em diversas questões, tais como: aprendizagem, praticidade e rapidez, conhecimento do novo, comunicação e acesso à informação, realização de pesquisas e acesso a notícias e desenvolvimento de novas capacidades.

Apesar do uso constante desses dispositivos pelos idosos, metade deles (50\%) nunca haviam utilizado uma m-learning app. Entretanto, 25 dos respondentes $(89,28 \%)$ 
VII Congresso Brasileiro de Informática na Educação (CBIE 2018)

Anais do XXIX Simpósio Brasileiro de Informática na Educação (SBIE 2018)

disseram acreditar que os dispositivos móveis podem auxiliar no processo de ensinoaprendizagem, e portanto, como um meio para viabilizar mais suporte para educação. Os dados indicaram que a maioria dos partipantes (96\%) têm interesse em usar m-learning app. Os participantes também responderam que gostariam de aprender sobre diversos assuntos com o uso de m-learning apps, conforme segue: (i) Agricultura (3,57\%); (ii) Assuntos religiosos (3,57\%); (iii) Conhecimentos gerais ou diferentes áreas (53,57\%); (iv) História (7,14\%); (v) Outras línguas (21,42\%); (vi) Português $(3,57 \%)$; (vii) Uso do celular, tecnologias ou computação $(7,14 \%)$.

A partir de uma análise geral dos dados, é possível afirmar que não há uma distinção clara entre os assuntos de interesse de mulheres e homens, bem como em relação à idade da pessoa. Eventualmente, o interesse em Tecnologia e Computação foi prioritariamente dos homens, enquanto o interesse por História foi específico das mulheres. Entretanto, esses dados são insuficientes para formular ou comprovar conclusões mais assertivas.

Com respeito às m-learning apps, segundo a pesquisa, é necessário que elas apresentem características específicas. Dentre elas, destacam-se: de que ela seja intuitiva $(21,42 \%)$ e colorida $(17,85 \%)$. A Figura 3 sumariza as características manifestadas pelos participantes para uma m-learning app.

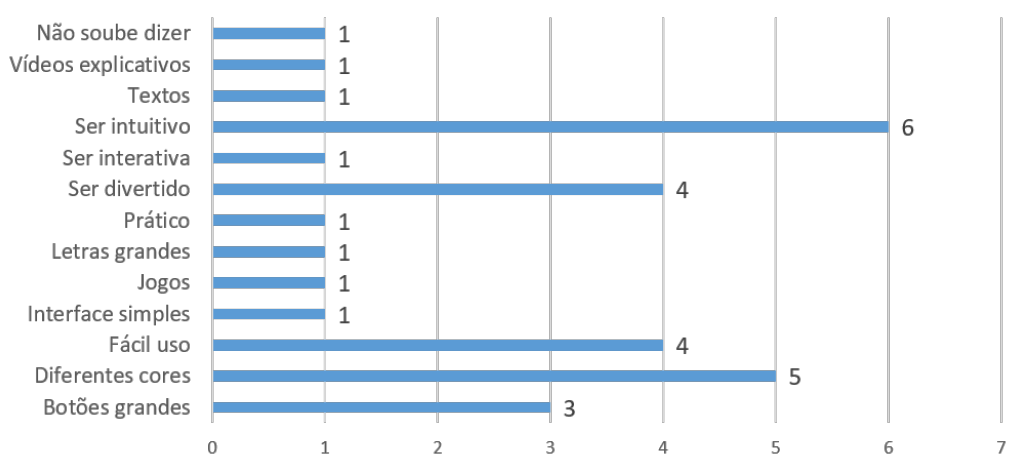

\section{Figura 3. Funcionalidades/características para uma aplicação de aprendizagem móvel}

Analisando as características descritas pelos participantes e a idade de cada um deles, também não é possível afirmar que pessoas mais velhas têm interesses específicos. Os resultados mostraram que tanto os idosos mais novos quanto os mais velhos possuem, por exemplo, interesse por botões e letras grandes, bem como por aplicativos coloridos, fáceis e que lidem com gameficação.

\subsection{Entrevista realizada com os especialistas}

A fim de complementar os dados obtidos com o questionário, também foram realizadas entrevistas semi-estruturadas com dois especialistas (um homem e uma mulher) no ensino de idosos. O homem possuía três anos de experiência com este tipo de atividade e a mulher dois anos de experiência.

Segundo os especialistas, o curso de Práticas de Tablets e Smartphones tem início com atividades simples, evoluindo para tarefas mais complexas posteriormente. Os idosos aprendem desde como procurar e salvar um contato até instalação de aplicativos, nuvem e 
VII Congresso Brasileiro de Informática na Educação (CBIE 2018)

Anais do XXIX Simpósio Brasileiro de Informática na Educação (SBIE 2018)

configurações. Dentre as atividades destaca-se o uso do Whatsapp, Facebook, Instagram, além de aplicativos do Sistema Unico de Saúde (SUS) do Brasil e de aplicativos nativos (e.g., Maps). Ambos os entrevistados observaram que os idosos têm mais interesse em aprender sobre o uso do Whatsapp e Facebook.

Quanto às dificuldades enfrentadas pelos idosos ao utilizar as aplicações móveis, os especialistas destacaram: (i) tela sensível ao toque; (ii) tamanho da tela; (iii) entender o que o aplicativo está querendo informar; (iv) receber feedback do aplicativo; (v) Fat finger (Dedo gordo); (vi) esquecer o significado de algum ícone ou funcionalidade do sistema; (vii) falta de prática, (viii) lidar com o teclado local; (iv) tocar no campo para entrada de texto e digitar. Os dois especialistas também acreditam que os idosos teriam interesse em utilizar m-learning apps. Segundo eles, este público teria interesse em aprender sobre inglês ou outras línguas, por meio de jogos que estimulassem a parte cognitiva ou matemática envolvendo o raciocínio lógico.

A interface, nas m-learning apps para idosos, deveria apresentar como principais características: (i) ser simples, intuitiva e com poucas funcionalidades; (ii) apresentar feedback adequado ao usuário; (iii) disponibilizar um passo-a-passo (guia) ao usuário; (iv) possuir controles de acessibilidade; (v) contraste; (vi) possuir fonte maior; e (vii) necessitar de poucos passos durante a interação.

Ainda de acordo com os especialistas, entre as funcionalidades desejáveis e que potencializariam a atenção e motivação para o aprendizado, que deveriam estar presentes nas m-learning apps orientadas para idosos, destacam-se: (i) apresentar sistemas de recompensa, (ii) ter uma proposta de fazer atividades com pontuação, (iii) possuir um compartilhamento interno com amigos sobre a pontuação ou algo que estimule a competição.

Fazendo uma comparação entre os dados tabulados no questionário e os dados da entrevista, é possível perceber semelhanças entre eles. Nota-se que algumas dificuldades descritas pelos especialistas poderiam ser solucionadas se a aplicação fosse desenvolvida com base na necessidade dos usuários. A dificuldade com o tamanho da tela, por exemplo, poderia ser solucionada a partir do desenvolvimento de uma aplicação com letras e botões maiores. $\mathrm{Ou}$, ainda, a dificuldade com o entendimento sobre determinada m-learning app poderia ser minimizada com a implementação de uma aplicação com interface mais intuitiva e de fácil uso. De fato, os dados ressaltaram a necessidade dos desenvolvedores terem maior conhecimento sobre seu público alvo, de modo a projetarem as aplicações levando em conta as características específicas dos seus usuários.

Com base nos dados coletados a partir do questionário e da entrevista, foi proposto um protótipo de m-learning app voltada ao contexto de usuários idosos, a Crossword Learning, apresentada na próxima seção.

\section{Uma m-learning app: Crossword Learning}

A Crossword Learning é uma m-learning app, proposta com base nos dados do questionário e das entrevistas realizadas. A aplicação tem como público-alvo usuários idosos, apresenta uma interface simples, intuitiva, com menus de acessibilidade, e aborda o ensino de temas variados, tais como História, Geografia, Inglês, Português, dentre outros. A escolha pelos conteúdos foi feita a partir dos dados do questionário, visto que os participantes mostraram interesse em áreas diversas. 
VII Congresso Brasileiro de Informática na Educação (CBIE 2018)

Anais do XXIX Simpósio Brasileiro de Informática na Educação (SBIE 2018)

Na Crossword Learning são definidos níveis de dificuldade (fácil, médio e difícil), os quais foram definidos para atender à possibilidade de adaptação de perfis distintos de idosos ao usarem a aplicação. O foco não é o jogo em si, mas sim a utilização de palavras cruzadas no ensino e aprendizado. Assim, o usuário pode aprender sobre diferentes assuntos, sempre tendo um contexto para cada uma das atividades (Figura 4).

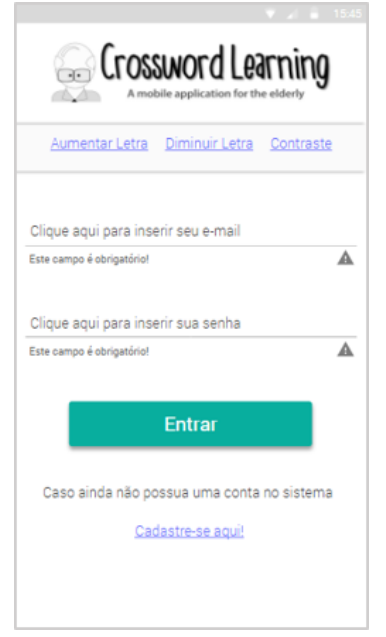

(a) Tela inicial

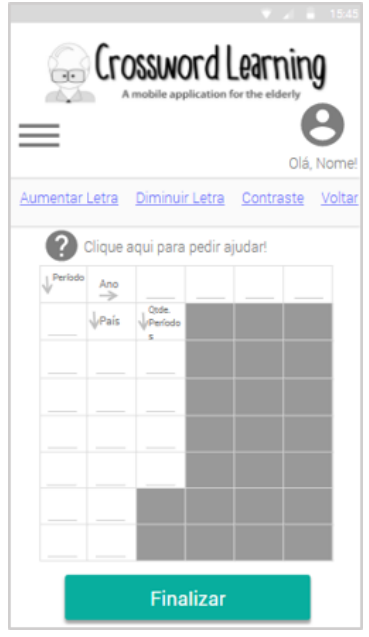

(b) Tela de palavra cruzada

\section{Figura 4. Interface da m-learning app "Crossword Learning"}

Ao realizar as atividades propostas (palavras cruzadas), o usuário ganha pontos e evolui para níveis mais complexos. O usuário pode ter acesso a sua pontuação e ter um feedback que representa o quanto ele aprendeu durante o uso da m-learning app, baseando-se em dados estatísticos. Além disso, a aplicação permite que o usuário participe de competições e compartilhe seus resultados dentro de sua rede interna de amigos.

Pode-se destacar, ainda, os seguintes recursos da aplicação: (i) apresentação de vários conteúdos por meio de vídeo, texto ou áudio; (ii) registro de pessoas em uma rede social interna; (iii) compartilhamento de resultados das atividades/fases com a sua rede de amigos; e (iv) monitoramento do nível de aprendizagem por meio do ranking pessoal. É importante ressaltar que a escolha pelas funcionalidades e recursos foi realizada com base nos questionários e nas entrevistas realizadas, conforme apresentados anteriormente.

A partir da definição dos requisitos gerais da aplicação, o protótipo foi estruturado no Justinmind [Farrell-Vinary 2011], software para prototipação de aplicações web e móveis. Após o desenvolvimento do protótipo, foram realizados testes com usuários e avaliações heurísticas, a fim de verificar questões de acessibilidade e usabilidade. Uma descrição mais detalhada da aplicação e resultados dos testes realizados com idosos podem ser encontrados em [Oliveira et al. 2018].

\section{Conclusão e trabalhos futuros}

Neste artigo foi apresentado um estudo sobre o uso de dispositivos móveis e aplicações de aprendizagem móvel (m-learning apps) no contexto de usuários idosos. Os dados, obtidos por meio de questionários e entrevistas, foram apresentados e analisados de modo a delinear o perfil de usuários aprendizes idosos. 
VII Congresso Brasileiro de Informática na Educação (CBIE 2018)

Anais do XXIX Simpósio Brasileiro de Informática na Educação (SBIE 2018)

De modo geral, observou-se que os idosos têm interesse em aprender sobre vários assuntos e fazem uso de aplicações móveis diariamente. Entretanto, a maioria deles nunca teve contato, de fato, com m-learning apps. Os resultados também indicaram a importância de se desenvolver m-learning apps com características específicas, dando especial atenção à necessidade de serem intuitivas, coloridas, de fácil uso e divertidas (com menos seriedade), entre outros aspectos. Além disso, foi proposta a m-learning app denominada Crossword Learning, aplicação que trabalha com aspectos de ensino e aprendizagem dos idosos por meio de palavras cruzadas. A Crossword Learning foi desenvolvida com base no perfil dos usuários idosos e contemplou as características indicadas pelos participantes dos questionários e das entrevistas.

Como trabalhos futuros, novas questões devem ser inseridas ao questionário e à entrevista, a fim de verificar as barreiras de acessibilidade e usabilidade enfrentadas por esses usuários. Fazem-se necessárias, ainda, novas aplicações do questionário e da entrevista, de modo que a amostra de usuários seja maior, inclusive com a possibilidade de análises estatísticas sobre o tema. O desenvolvimento, avaliação e aplicação da Crossword Learning em cenários reais de ensino e aprendizagem também está previsto.

Ressalta-se, por fim, que os resultados desse estudo têm sido utilizados no estabelecimento de um conjunto de diretrizes de acessibilidade que auxilie no desenvolvimento de m-learning apps com foco em usuários idosos.

\section{Agradecimentos}

Este estudo foi financiado pela Universidade de São Paulo (USP) e pelas agências de fomento brasileiras: Coordenação de Aperfeiçoamento de Pessoal de Nível Superior Brasil (CAPES) - Código de Financiamento 001/Procad 071/2013, CNPq e FAPESP.

\section{Referências}

Boulos, M. N. K., Brewer, A. C., Karimkhani, C., Buller, D. B., and Dellavalle, R. P. (2014). Mobile medical and health apps: state of the art, concerns, regulatory control and certification. Online journal of public health informatics, 5(3):229.

Brasil (2003). Estatuto do idoso. http://www.planalto.gov.br/ccivil_03/leis/2003/L10.741.htm.

Camarano, A. A., Kanso, S., and Mello, J. L. (2004). Como vive o idoso brasileiro. Os novos idosos brasileiros: muito além dos, 60(1):25-73.

Dix, A., Finlay, J. E., Abowd, G. D., and Beale, R. (2003). Human-Computer Interaction (3rd Edition). Prentice-Hall, Inc., Upper Saddle River, NJ, USA.

Farrell-Vinary, P. (2011). Justinmind. ACM SIGSOFT Software Engineering Notes, 36(3):34-35.

Gerhardt, T. E. and Souza, A. C. (2009). Aspectos teóricos e conceituais. Métodos de Pesquisa. Porto Alegre: Editora da UFRGS.

Gil, A. C. (2002). Como elaborar projetos de pesquisa. São Paulo, 5(61):16-17.

Hanson, V. L. (2010). Influencing technology adoption by older adults. Interacting with Computers, 22(6):502 - 509. Special Issue on Inclusion and Interaction: Designing Interaction for Inclusive Populations. 
VII Congresso Brasileiro de Informática na Educação (CBIE 2018)

Anais do XXIX Simpósio Brasileiro de Informática na Educação (SBIE 2018)

IBGE (2010). Instituto brasileiro de geografia e estatística - censo demográfico. https://www.ibge.gov.br/estatisticas-novoportal/sociais/populacao.html.

ISO, I. (2013). 20282-2:2013. Usability of consumer products and products for public use.

Kraut, R. (2013). Policy guidelines for mobile learning. UNESCO.

Kukulska-Hulme, A. and Sharples, M. (2016). Waypoints along learning journeys in a mobile world. Sustaining mobile learning: theory, research and practice, pages 43-56.

Kurniawan, S., Mahmud, M., and Nugroho, Y. (2006). A study of the use of mobile phones by older persons. In CHI'O6 extended abstracts on Human factors in computing systems, pages 989-994. ACM.

Lazar, J., Feng, J. H., and Hochheiser, H. (2010). Research methods in human-computer interaction. John Wiley \& Sons, United Kingdom, 1 edition.

Marli, M. (2017). Expectativa de vida do brasileiro sobe para 75,8 anos. https://agenciadenoticias.ibge.gov.br/agencia-noticias/2012-agencia-denoticias/noticias/18469-expectativa-de-vida-do-brasileiro-sobe-para-75-8-anos.html.

Oliveira, C. D., Fortes, R. P. M., and Barbosa, E. F. (2018). An analysis of crossword learning: a mobile application for the elderly. In 20th International Conference on Human-Computer Interaction/HCI International. Springer. Las Vegas, Nevada USA, 15-20 July.

O’Malley, C., Vavoula, G., Glew, J., Taylor, J., Sharples, M., Lefrere, P., Lonsdale, P., Naismith, L., and Waycott, J. (2005). Guidelines for learning/teaching/tutoring in a mobile environment.

Organization, W. H. et al. (2016). World Health Statistics 2016: Monitoring Health for the Sustainable Development Goals (SDGs). World Health Organization.

Sarrab, M. (2015). M-learning in education: Omani undergraduate students perspective. Procedia-Social and Behavioral Sciences, 176:834-839.

Sharples, M., Arnedillo-Sánchez, I., Milrad, M., and Vavoula, G. (2009). Mobile learning. In Technology-enhanced learning, pages 233-249. Springer.

Silva, B. M., Rodrigues, J. J., de la Torre Díez, I., López-Coronado, M., and Saleem, K. (2015). Mobile-health: A review of current state in 2015. Journal of biomedical informatics, 56:265-272.

W3C (2017). Mobile accessibility at w3c. https://www.w3.org/WAI/standardsguidelines/mobile/.

Wang, Y.-S., Wu, M.-C., and Wang, H.-Y. (2009). Investigating the determinants and age and gender differences in the acceptance of mobile learning. British journal of educational technology, 40(1):92-118.

Whitbourne, S. K. (2008). Adult development and aging: Biopsychosocial perspectives. John Wiley \& Sons.

Zimmer, M., Trombetta, M., Biduski, D., De Marchi, A., and Colussi, E. (2013). Um aplicativo móvel para treino de memória em idosos: desenvolvimento e avaliação. Anais Tise, pages 715-18. 